\title{
A Simulation Study of Combined Routing and Contention Resolution Algorithms in Connection-Oriented OPS Network Scenario
}

\author{
M. Klinkowski, D. Careglio, X. Masip-Bruin, S. Spadaro, S. Sanchez-López, J. Solé-Pareta \\ Advanced Broadband Communications Centre (CCABA) \\ Universitat Politècnica de Catalunya (UPC), \\ Jordi Girona, 1-3, 08034 Barcelona, Catalunya, Spain \\ e-mail: \{mklinkow, careglio, xmasip,sergio, pareta\}@ac.upc.es, spadaro@tsc.upc.es
}

\begin{abstract}
Connection-oriented optical packet-switched network integrates MPLS and OPS layers to exploit the best of electronic and optical technologies. In particular, routing components use enhanced routing algorithms to compute the paths; forwarding components examine the labels of incoming packets to determine the next hop and contention resolution algorithms to solve packets contention; finally packets are physically transferred to the output interface, properly chosen by the forwarding component, using optics. In this paper we describe the details of such a network scenario, identifying some problems and suggesting different routing and contention resolution algorithms. The resulting network is evaluated by simulation in order to obtain network performance characteristics for suitable combined selection of these algorithms.
\end{abstract}

Keywords: optical packet networks, routing algorithms, contention resolution algorithms

\section{INTRODUCTION}

The networking trend is currently experiencing a migration towards a DWDM network. In this paper we focus on an optical network integrating a connection-oriented layer like MPLS (Multi-Protocol Label Switching) and the OPS (Optical Packet Switching) layer. Two concurrent mechanisms operate in such a network, namely the routing algorithm and the contention resolution algorithm. The former operates at network level to manage the paths between nodes and requires the network state information. There are several proposals in recent literature including TE enhancements to support QoS requirements (see for instance [1] [2]). Main proposals address the routing problem considering source routing because it allows the network to more efficiently distribute and control the traffic flows and avoids the scalability concerns of centralised routing. The latter operates at node level to find a conflict-free schedule of packets and requires the node state information. The technology limitation of optical queuing motivates significant research efforts in recent years dealing with the design of simple and efficient contention resolution policies for single optical packet switches (see for instance [3] [4] [5]). In the proposed scenario, the routing objective is to help the contention resolution algorithm of reducing the packet losses at the nodes balancing the link loads and avoiding queuing congestion. In this paper, we study and evaluate the entire network scenario combining the routing and contention resolution algorithms in a connectionoriented optical packet-switched network. It allows to find performance characteristics related to the real network as well as to investigate network behaviour in order to select suitable combination of these algorithms.

The rest of the paper is organised as follows. In Section 2 the details of the network are discussed. In Section 3 we suggest two enhanced source-based routing algorithms. Then in Section 4 we review two different contention resolution algorithms. Therefore, the resulting network is evaluated by simulation in Section 5 . Finally, Section 6 concludes the paper.

\section{NETWORK SCENARIO}

The network scenario consists of two different layers, namely the MPLS-like layer which provides the tools for effective TE management and the OPS layer which provides the transparent all-optical equipments capable to carry data-centric traffic at huge bit-rates.

The resulting connection-oriented, OPS network (see Fig. 1) comprises several nodes connected in a mesh topology. In this study we assume that the edge and core nodes are capable of switching asynchronous variable length packets (AVLP), which easily match with IP packets [4] coming from legacy networks. The edge nodes are in charge of collecting incoming data from legacy networks and arrange them into optical packets as well as managing the Optical Label Switched Paths (O-LSPs). The core nodes are in charge of switching the packets in the network according to the forwarding table. It is important to notice that inside OPS network only the packet header (label) is converted from optical to electrical format.

This work has been partially funded by the MCYT (Spanish Ministry of Science and Technology) under contract FEDERTIC2002-04344-C02-02 and the CIRIT (Catalan Research Council) under contract 2001-SGR00226. 


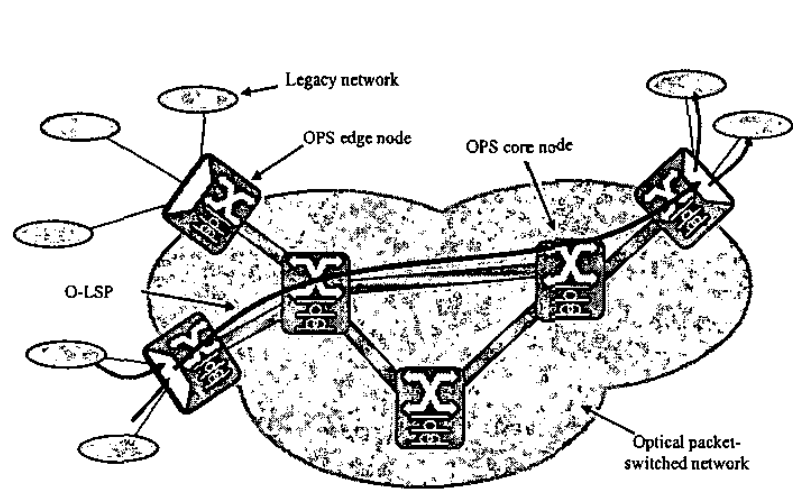

Figure 1. MPLS over OPS scenario.

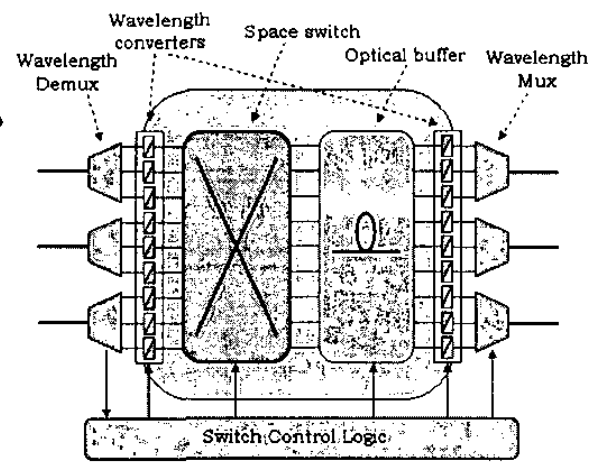

Figure 2. Node architecture with output feed-forward queuing configuration.

\section{ENHANCED ROUTING ALGORITHMS}

The main goal of TE is to optimize resource utilization and network performance. One aspect to be considered to do TE efficiently is the routing algorithm. The main function of any routing algorithm is to compute the paths. However, several factors such as the path signalling and the network state information reliability strongly interacts with such a function [2]. In fact, routing decisions performed by the source (edge) node will be optimal as long as this information perfectly represents the actual network state. This is impossible in real networks where several factors, such as non-negligible propagation delay of network state information, affect the precision of the global network state information [1]. Thus, the path selection process may be performed based on inaccurate network state information which implies substantial performance degradation (i.e. high packet losses at the nodes). Two enhanced source-based routing mechanisms, which include the network state information inaccuracy as a metric to select the paths in the proposed network, are discussed below.

The enhanced routing algorithms are based on the BYPASS Based Routing (BBR) mechanism proposed in [6] for IP/MPLS networks. It aims of improving the network performance when selecting explicit paths based on inaccurate network state information. The main concept of the BBR mechanism is the dynamic bypass concept, which is based on computing more than one feasible route to reach the destination. BBR instructs the edge node to compute both the working route and a certain number of bypass-paths: paths that bypass those links (called Obstruct-Sensitive Links, OSL) that potentially might not be able to cope with the incoming LSP demand requirements. Different routing algorithms can be inferred from the BBR mechanism. In this paper we consider:

- Shortest-Obstruct-Sensitive Path (SOSP) algorithm [6] which combines the BBR mechanism with the Dijkstra's algorithm computing the shortest path among all the paths that have the minimum number of OSLs.

- Balanced-Obstruct-Sensitive Path (BOSP) algorithm [6] which has the aim of balancing the path length and the residual bandwidth. It is based on minimizing a new parameter $\left(F_{p}\right)$, which represents the relation between the maximum residual bandwidth $b_{r}{ }^{i}$ and the number of hops $n$ along a path, so balancing the network load and the network occupancy, according to the expression:

$$
F_{p}=n \times \max \left(\frac{1}{b_{r}^{i}}\right) \quad i=1, \ldots, n
$$

Note that the main concept introduced in the BBR mechanisms has been already proposed in IP/MPLS networks. The scope here is to translate this concept in the proposed connection-oriented, OPS scenario.

\section{CONTENTION RESOLUTION ALGORITHMS}

Contention resolution algorithms are implemented at the nodes to solve the situation when two or more packets simultaneously want to access the same output interface. In this paper, we consider a general OPS node architecture with full connectivity and wavelength conversion as shows in Fig. 2, acting as an output queuing switch. The switch uses a feed-forward queuing configuration and the optical degenerate buffer [3] made by $B$ delay lines with wavelength multiplexing capability. The delays provided are linearly increasing with a basic delay unit $D$.

Some recent works show that integrating the O-LSPs status in the contention resolution schemes may further improve the performance of OPS nodes [5]. In this environment, during the setup procedure, the O-LSP is assigned to a given output wavelength at each node (LSP-to-wavelength assignment procedure); consequently, the packets belonging to that O-LSP are always switched to the same output wavelength. The node maintains this 
assignment over the whole connection lifetime except when congestion arises on the output wavelength; in this case, the node switch control executes a dynamic algorithm that temporarily moves the path to another output wavelength. The most interesting dynamic algorithms are:

- Empty Queue Wavelength Selection (EQWS) which is able to full exploit the queuing space available in the switching matrix by taking advantage of the presence of a group of O-LSPs incoming on the same input wavelength and forwarded to the same output wavelength (we call this situation optimal allocation).

- Minimum Queue Wavelength Selection (MQWS) which aims at reducing the packet delay as much as possible. When a packet following an O-LSP arrives to a congested queue, the algorithm looks for the wavelength of the same fibre that has the shortest waiting queue.

\section{PERFORMANCE EVALUATION}

The aim of this paper is to compare the results in terms of network performance when applying SOSP and BOSP algorithms as routing algorithms and EQWS and MQWS as contention resolution algorithms. Therefore, a set of simulations has been carried out to evaluate the performance of all possible combinations (i.e. SOSP/EQWS, SOSP/MQWS, BOSP/EQWS, and BOSP/MQWS). In order to have a benchmark to compare improvements to, we also consider the "classical" WSP (Widest Shortest Path [1]) routing algorithm (i.e. WSP/EQWS and WSP/MQWS combinations). It has to be underlined again that in the considered connection-oriented, OPS network, better performance means low Packet Loss Probability (PLP) at the nodes.

\subsection{Simulation scenario}

Simulations have been carried out over the network topology shown in Fig. 3, which consists of 18 edge nodes and $28 \mathrm{bi}$-directional links with 16 wavelengths at $2.5 \mathrm{Gbit} / \mathrm{s}$ each. The links have the same length of $500 \mathrm{~km}$.

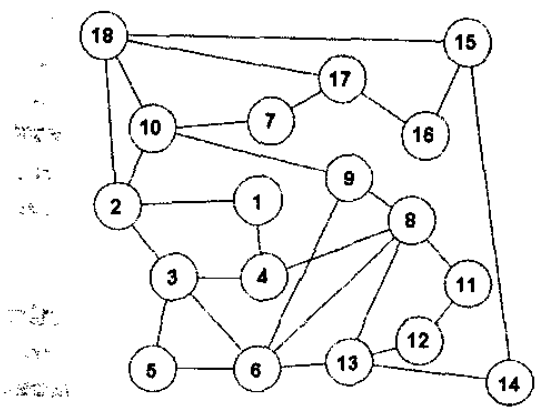

Figure 3. Network Topology. Every simulation set up 1700 O-LSPs according to the selected routing algorithm. The source and destination nodes of the O-LSPs were uniformly selected. The packet traffic within each O-LSP has been generated by a source implemented using a self-similar traffic model generating IP packets. Each source has been modeled as a superposition of 32 strictly alternating ON/OFF Pareto distribution sources with $\alpha=1.2$, which leads to an Hurst parameter of $H=0.9$. The IP packets have been generated according to the Internet packet size distribution. The FDLs' granularity of the OPS nodes was assumed equal to the mean packet size ( 400 bytes), which provides the best packet losses as demonstrated in [5]. The length of the FDLs is the same for all nodes and set to $B=4$.

\subsection{Simulation results}

Figure 4 shows the packet loss probability (PLP) of each node comparing the WSP, SOSP, and BOSP routings combined with the MQWS algorithm. It can be seen that adopting WSP/MQWS, the nodes experience very different PLPs, ranging from $2.8 \times 10^{-3}$ at node 3 to $10^{-7}$ at node 1 . Otherwise, BOSP/MQWS achieves the best results since all nodes lose less than $7 \times 10^{-5}$ packets. SOSP/MQWS shows intermediate values between BOSP/MQWS and WSP/MQWS performances.

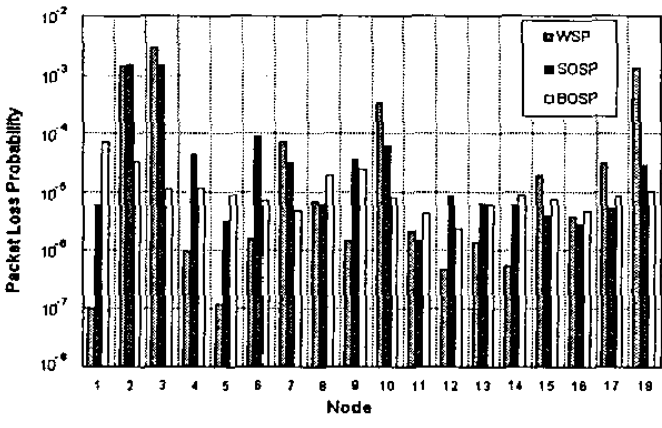

Figure 4. Packet loss probability combining the routing mechanisms with the MQWS algorithm.

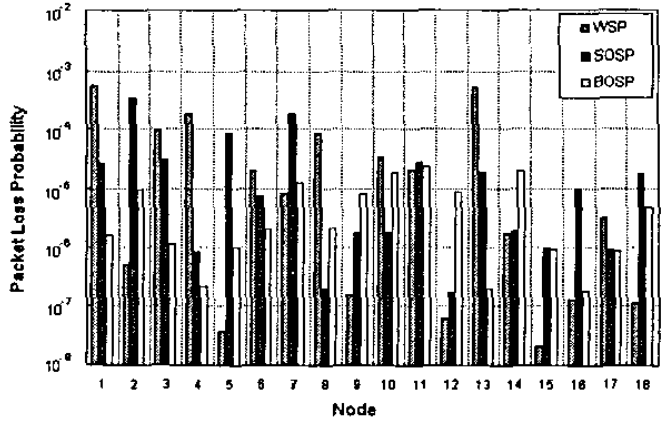

Figure 5. Packet loss probability combining the routing mechanisms with the EQWS algorithm. 
Figure 5 shows the packet loss probability (PLP) of each node comparing the WSP, SOSP, and BOSP routings using the EQWS algorithm. Again, the BOSP/EQWS algorithm presents the best results, while the SOSP/EQWS shows intermediate values between BOSP/EQWS and WSP/EQWS performances.

A cross comparison between the BOSP/EQWS and BOSP/MQWS combinations shows that the former presents better overall network performance since almost all nodes obtain lower PLPs. Indeed, the average PLP (the sum of all PLPs divided by the number of nodes) is $1.41 \times 10^{-5}$ for the BOSP/MQWS case while it is $6.51 \times$ $10^{-6}$ for the BOSP/EQWS case (see table 1).

Table 1. Average PLP comparison.

\begin{tabular}{l|l|l|l} 
& WSP & SOSP & BOSP \\
\hline MQWS & $3.38 \times 10^{-4}$ & $1.82 \times 10^{-4}$ & $1.41 \times 10^{-5}$ \\
\hline EQWS & $8.54 \times 10^{-5}$ & $4.25 \times 10^{-5}$ & $6.51 \times 10^{-6}$
\end{tabular}

Table 2. Fairness Index comparison.

\begin{tabular}{l|l|l|l} 
& WSP & SOSP & BOSP \\
\hline MQWS & 27872 & 1008 & 31 \\
\hline EQWS & 27542 & 2021 & 127
\end{tabular}

Table 2 compares the fairness index of all possible algorithm combinations. The fairness index is defined as the ratio between the maximum and the minimum PLP experienced by the nodes. For instance, using SOSP/MQWS routing, node 2 shows the maximum PLP $\left(1.49 \times 10^{-3}\right)$ and node 11 the minimum one $\left(1.48 \times 10^{-6}\right)$. The ratio between these values is the fairness index (1008). Given that lower values n:ean higher network balancing, BOSP/MQWS presents the best fairness degree.

\section{CONCLUSIONS}

In this paper we have presented a connection-oriented, OPS network able to meet the current service and performance requirements combining the routing and the contention resolution mechanisms. For the routing problem, we have applied two algorithms (SOSP and BOSP) inferred from the BYPASS Based Routing mechanism to optimise the network resource utilisation and reduce the impact of the routing inaccuracy problem. For the contention resolution problem, we have applied two different algorithms (MQWS and EQWS) capable of switching AVLP packet and solving the packet contentions taking into account the status of the O-LSPs.

The simulation results show that, whatever contention resolution algorithm is used, the SOSP and BOSP routing algorithms substantially improves the network performance decreasing the packet loss probability compared to the "classical" WSP algorithm. In particular, the combination BOSP/MQWS achieves a very high degree of balancing between the performances of the nodes (better fairness) while BOSP/EQWS presents the best overall network performance.

\section{ACKNOWLEDGMENTS}

The authors would like to thank Prof. Franco Callegati and Dr. Walter Cerroni for their useful comments and suggestions.

\section{REFERENCES}

[1] R.A. Guerin, A. Orda :QoS routing in networks with inaccurate information: theory and algorithms, IEEE/ACM Transactions on Networking, vol. 7, no. 3, Jun. 1999, pp. 350-364.

[2] X. Xiao:Traffic engineering with MPLS in the Internet, IEEE Network, vol. 14, no. 2, Mar. 2000, pp. 2833.

[3] D.K. Hunter, M.C. Chia, I. Andonovic: Buffering in optical packet switches, IEEE/OSA Journal of Lightwave Technology, vol. 16, no. 2, Dec. 1998, pp. 2081-2094.

[4] L. Tanc vski, S. Yegnanarayanan, G. Castanon, L. Tamil, F. Masetti, T. McDermott :Optical routing of asynchronous, variable length packets, IEEE Journal on Selected Areas in Communications, vol. 18, no. 10, Oct. 2000, pp. 2084-2093.

[5] F. Callegati, W. Cerroni, C. Raffaelli, P. Zaffoni, Dynamic wavelength assignment in MPLS optical packet switches, Optical Network Magazine, vol. 4, no. 5, Sep. 2003, pp. 41-51.

[6] X. Masip-Bruin, S. Sanchez-López, J. Solé-Pareta, J. Domingo-Pascual :QoS routing algorithms under inaccurate routing information for bandwidth constrained applications, in Proc. IEEE 2003 International Conference on Communications (ICC 2003), Anchorage, AL, May. 2003, pp. 1743-1748. 